\title{
Research on Meat Product Traceability System Based on Two Dimensional Code
}

\author{
Yanbai Wang and Lu Tan ${ }^{*}$ \\ ${ }^{1}$ Changchun University Changchun Jilin China \\ ${ }^{*}$ Corresponding author
}

\begin{abstract}
Meat products safety related to the national economy and the people's livelihood, the relationship between the national health. Meat products in the breeding, slaughter, storage and sales process of real-time monitoring and the establishment of meat products safety traceability system is the main means to improve the status of meat products. Objective to improve the safety of meat products, meat products through the establishment of two-dimensional code technology based on traceability management system, so that consumers understand the meat products in the breeding, slaughtering, storage and sales process of the right to know, to increase the transparency of information of meat products in the whole process flow of.
\end{abstract}

Keywords-two dimensional code; origin ; meat product

\section{INTRODUCTION}

In recent years, domestic and foreign meat products safety (food poisoning, mad cow disease, foot-and-mouth disease, avian influenza, PRRS and other livestock epidemic and clenbuterol, artificial feeding and other hormone) crisis occurred frequently, seriously affecting people's health. Has aroused the concern of the community on the safety of meat products, some consumers even formed a anxiety. Therefore, how to ensure the safety of meat products, the implementation of effective processing of meat products traceability system, to achieve the responsibility mechanism of offenders, has become a very urgent global issues. The traceability of meat products is more and more concerned and valued, which is regarded as an important means to manage and control the safety of meat products. However, there are serious problems in the safety and quality of meat products, and it is urgent to study the technology of the meat industry. Feeding is beef cattle products throughout the production process (feeding, slaughter, processing, warehousing, logistics, sales) in the longest cycle of a link, but also the quality of beef cattle is the main link. Therefore, it is necessary to raise the stage of information acquisition and recording accurately, and effective management in the breeding process data submitted to the management system, in the end product key control points of beef cattle breeding by inverse tracing information. Starting from the source, it is very necessary and urgent to study the information collection of the key control points and the product quality and safety traceability of beef cattle feeding.

With the development of barcode technology, twodimensional code with its irreplaceable advantage has become an important part of information work, the use of twodimensional code has a large storage capacity, high security, can spread true, traceability, abrasion resistance, the advantages of backup etc., suitable for use in meat products traceability.

\section{TWO-DIMENSIONAL CODE}

Two dimensional bar code / two-dimensional code is a kind of special geometric figure according to certain rules in the plane (two-dimensional direction) distribution of black and white graphics data symbols information; In coding, the clever use of a computer-based internal logic " 0 " and "1" bit stream concept, using a plurality of corresponding binary geometric shapes to represent the text value of information, through the image input device or optical scanning equipment automatic recognition to achieve automatic information processing: it has some common bar code technology each code system has its specific character set; each character occupies a certain width; has a calibration function etc.. At the same time, it also has the function of automatic identification of non peer information, and the change point of processing graphics rotation.

\section{A. Working Principle of Two-dimensional Code}

In coding, the clever use of a computer-based internal logic "0" and "1" bit stream concept, using a plurality of corresponding binary geometric shapes to represent the text value of information, through the image input device or optical scanning device to realize the automatic processing of information automatic recognition. In many kinds of twodimensional bar code, commonly used code is: Matrix MaxiCode, Data, Aztec, Code QR, Vericode, PDF417, Ultracode, Code 49, 16K Code, Code QR code is invented in 1994 by the Japanese DW company. QR from the English "Response Quick" abbreviation, namely the meaning of fast response, from the inventor hope QR code can let its content quickly be decoded. QR code is most common in Japan, South Korea, and Japan's most popular two-dimensional space bar code. But the security of two-dimensional code is also a challenge, with malware and viruses are becoming a stumbling block on the road to universal. The development and prevention of the abuse of two-dimensional code is becoming an urgent problem to be solved.

Each code has its own set of characters, each character has a certain width, with a certain check function, etc.. At the same time, it also has the characteristics of automatic recognition of non peer information and processing graphics rotation change.

Two dimensional code is a more advanced than one dimensional code bar code format. One dimensional code can 
only express information in one direction (usually horizontal direction), and the two-dimensional code can store information in horizontal and vertical direction. One dimensional code can only be composed of numbers and letters, and two-dimensional code can store information, such as Chinese characters, numbers and pictures, so the application area of twodimensional code is much wider.

Two dimensional bar code and two-dimensional code can be divided into stacked type / row type two-dimensional bar code and matrix two-dimensional bar code. Stacked / stacked barcode form is composed of multi line cutting short stacked one-dimensional bar code and composition; matrix twodimensional bar code in the form of a matrix, the matrix elements corresponding position with the "point" binary "1", with "empty" binary "0", "point" and "empty row code. The principle of two-dimensional code can be based on the principle of two-dimensional code matrix and determinant of two-dimensional code to tell.

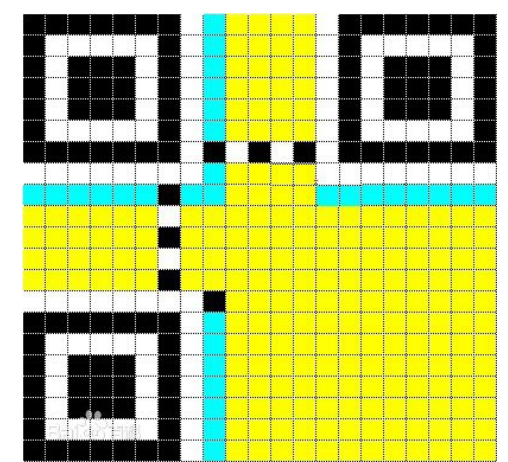

FIGURE I. MATRIX TWO-DIMENSIONAL CODE PRINCIPLE

\section{B. Characteristics of Two-dimensional Codes}

1) High density encoding, large information capacity, can accommodate up to 1850 letters or 2710 digits or 1108 bytes, or more than and 500 Chinese characters, dozens of times higher than ordinary bar code information capacity.

2) Encoding a wide range: the barcode can take pictures, text, voice, signature and fingerprint information can be digitized using bar code encoding, expressed; can be expressed in multiple languages; can represent image data.

3) Strong fault tolerance, with error correcting function: the two-dimensional barcode local damage caused by perforation, dust, can still get the correct reading, damaged area of $50 \%$ can recover information.

4) Decoding reliability is high: it is more than ordinary bar code decoding error rate of two is much lower, bit error rate of not more than 10 million.

5) The introduction of encryption measures: confidentiality, security is good.

\section{Storage and Color of Two-dimensional Code}

Two-dimensional code can store a variety of information, including: Web site, business cards, text information, specific code. According to the application of information, and can be divided into:

\section{1) Online applications}

Such as web site and specific code, more online applications.

\section{2) Offline applications}

Such as text messages and business cards, more is the next line applications.

The two-dimensional code we usually see, most software are black, but in fact the color of the two-dimensional code generation technology is not complicated, and many young people like, some web sites began to provide free online service to generate two-dimensional code color. The function of error correction based on two-dimensional bar code, twodimensional code even partially covered or lost, scanning equipment can still be identified the complete record information, the current generation tool of many "personality code", put some personality patterns and two-dimensional code synthesis, and can be personalized by scanning the twodimensional code recognition device.

\section{Two Dimensional Code Encryption Algorithm}

In order to improve the security of the two-dimensional code information, the DES algorithm and RSA algorithm are used to encrypt the two-dimensional code by the hybrid algorithm of the two encryption algorithms. DES encryption algorithm is the information packet, packet after the information re encryption. DES algorithm is fast, but the 56 bit key is not safe enough. RSA algorithm is a public key algorithm, it can produce 2 keys: one is the public key, and the other is the secret key. One key is used to encrypt, and the other is used for decryption. RSA algorithm is slow, but very safe. This study combines the advantages and disadvantages of the two, and designs the -RSA DES hybrid encryption algorithm. The use of DES algorithm for two-dimensional code text information encryption, and then RSA encryption key DES.

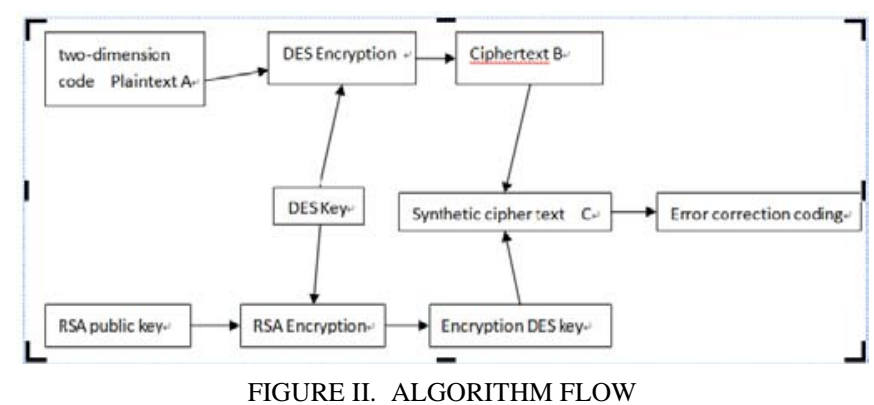

\section{Meat Product Traceability System Module DIVISION}

\section{A. Background Data Management Module}

This module is mainly operated by the administrator, on farms, slaughterhouses, distribution center (wholesale market) and data acquisition of the retail market for the collection, verification and storage, including the input information written to the information about the pig electronic tag, and the circulation node actively uploaded to the central database data source. 


\section{B. Data Statistical Analysis Module}

The main module in the background using some statistical algorithms and data mining based data acquisition module to data processing and analyzing, statistical results accurate and timely statistics and a certain breadth and depth of the analysis report, in addition, this module provides data source for the release of public module.

\section{Information Inquiry Module}

Consumers in the purchase of meat food, sweep using a mobile phone supermarkets, farms and other retail stores to provide two-dimensional code, we can get the meat sources, categories and shelf life of some of the basic information and the corresponding back yards, in addition to more detailed information, consumers can log on the system by virtue of the traceability code inquiry instruction detailed information about traceability system platform, associated with the meat back to the consumer.

\section{Information Release Public Module}

According to the results of the information statistical analysis module to form a statistical report, and in a friendly way to the consumer. At the same time will be based on the views and suggestions of consumers, will be the information of the consumer interest in real time to publish.

\section{E. Complaint Recommendation Module}

Consumers in the purchase of pork products, such as quality problems, complaints can be logged on to the system, the administrator will fill in the form according to consumer complaints, after verification, immediately make reasonable decisions, to ensure that consumers will minimize the losses. At the same time will also collect information on the user's comments and suggestions and other feedback, so that consumers eat at ease meat".

\section{F. User Management Module}

This module includes the administrator management and user management, the administrator is divided into super administrator and general manager, the general manager of the authority by the super administrator to distribution and its management, user registration information and audit related permissions set by the administrator.

\section{IMPLEMENTATION OF TRACEABILITY SYSTEM FOR MEAT PRODUCTS}

Meat traceability system management platform using EE JAVA development combined with HTML5, Spring, bootstrap and JS Angular and other technologies, select the Tomcat WEB Apache server, database using Server2008 SQL. Using B/S architecture to achieve the deployment of interaction, the use of HTTP to achieve communication between the user and the server protocol, the sensitive data using HTTPS for communication. This platform includes the comprehensive safety production information of meat products, and the management of these data, to provide security. Application of two-dimensional code technology allows people to easily and quickly check the information of meat products, standardize the production and operation of enterprises, but also to protect the rights and interests of consumers.

\section{CONCLUSION}

Meat safety has become an important problem in the world, with China's economic development, China has entered the era of meat safety. The two dimensional code technology, big data analysis technology and meat products traceability system combine to form a more perfect food safety monitoring system. The combination of the system allows people to understand the meat products of feeding, slaughter, processing, warehousing, logistics, sales and other aspects of data information, so that the general public to eat at ease meat products.

\section{ACKNOWLEDGMENT}

Thank you for the support of Jilin Provincial Department of Education (NATURAL SCIENCE) Research Plan [2013] No. 288

\section{REFERENCES}

[1] Liu Ying,Chen LiCheng . "Traceability" of the European Union and the United States: Introduction to beef production system[J]. Food Science,2003(8).

[2] Zhang Hui. Information security technology. People's Posts and Telecommunications Press,2013(3).

[3] China goods coding Center. Two dimensional bar code technology and Application,2007(7).

[4] Li Jia,Ma YuanJing,Jin Hua. Establishment of a new full traceability system for beef cattle production and cold fresh beef production[J] . Food science and technology.2014,39(01).

[5] Wang Shuo,Liu ShiHong,Zheng HuoGuo. Research and implementation of Xinjiang beef traceability system[J]. journal of Anhui agricultural sciences.2013(26). 